\title{
Energy Spot Price Models and Spread Options Pricing*
}

\author{
Samuel Hikspoors and Sebastian Jaimungal a \\ ${ }^{a}$ Department of Statistics and Mathematical Finance Program, University of Toronto, 100 St. George \\ Street, Toronto, Canada M5S 3G3
}

In this article, we construct forward price curves and value a class of two asset exchange options for energy commodities. We model the spot prices using an affine two-factor mean-reverting process with and without jumps. Within this modeling framework, we obtain closed form results for the forward prices in terms of elementary functions. Through measure changes induced by the forward price process, we further obtain closed form pricing equations for spread options on the forward prices. For completeness, we address both an Actuarial and a risk-neutral approach to the valuation problem. Finally, we provide a calibration procedure and calibrate our model to the NYMEX Light Sweet Crude Oil spot and futures data, allowing us to extract the implied market prices of risk for this commodity.

\section{Introduction}

The energy commodity markets are fundamentally different from the traditional financial security markets in several ways: Firstly, these markets lack the same level of liquidity that the majority of financial markets enjoy. Secondly, the storage costs of most commodities translate into peculiar price behavior; some commodities are extremely difficult to store or cannot be stored at all - electricity being a prime example. Thirdly, partly due to the structural issues surrounding energy price determination, electricity prices are typically exposed to very high volatility and to large shocks. Finally, commodity prices tend to have strong mean reverting trends. These stylized empirical facts are well documented in, for example, Clewlow and Strickland (2000), Carmona and Durrleman (2003), Eydeland and Wolyniec (2003) and Hull (2005).

The world wide energy commodities markets have created a need for a deeper quantitative understanding of energy derivatives pricing and hedging. We contribute to this program firstly by proposing a two-factor mean-reverting spot price process, both with and without a jump component, and secondly by carrying out the explicit valuation of spread options written on two forward prices. The spot price model is similar in spirit to the two-factor model proposed in Pilipovic (1997); however, in that work the second factor follows a geometric Brownian motion and, therefore, in the long run, the targeted mean blows up. Instead, we chose the mean-reverting level of the first factor to mean-revert to a second longrun mean. Our modeling framework can then be viewed as a perturbation on the standard one-factor mean-reverting approach. This is an appealing approach as the one-factor model has been extensively studied and approximately fits forward price curves. Adding a perturbation on top of this first order model allows us to correct some of the deficiencies of the one-factor model while maintaining tractability. In particular, the second factor perturbation does not change the stationary behavior that the one-factor model enjoys. We delegate the details of our purely diffusive model, and its relation and differences to the classical Pilipovic (1997) model, to Section 2.1 and of our jump-diffusion model to Section 4.1.

Much like the financial markets, energy markets are rife with derivative products. However, one product stands out among the many that are traded over the counter: spread options. These options provide the owner with the right to exchange a prespecified quantity of one asset for another, at a fixed cost. An

\footnotetext{
*The Natural Sciences and Engineering Research Council of Canada helped support this work.
} 
even more popular option is the spread option on forward prices which allows the holder to exchange two forward contracts, possibly with differing maturity dates, rather than the commodity. The holder of such an option receives at maturity $T$ a payoff of

$\varphi\left(F_{T, T_{1}}^{(1)}, F_{T, T_{2}}^{(2)}\right):=\max \left(F_{T, T_{1}}^{(1)}-\alpha F_{T, T_{2}}^{(2)}-K, 0\right)$.

It is well known that even when the commodity prices are modeled as geometric Brownian motions (GBMs), no closed form solution exists for $K \neq 0$. As such, we focus on the zero exchange cost case $K=0$ which we succeed in valuing in closed form under our two-factor mean-reversion modeling assumptions. Given our closed form solutions, the general case $K \neq 0$ can be valued using either Monte Carlo or PDE methods with our $K=0$ result acting as a control variate.

In a financial markets context, before proceeding to the valuation of derivatives, the objective measure is transformed to an equivalent risk-neutral measure. However, in the context of energy derivatives, due to the illiquidity issue, such a measure change is by no means necessary. Therefore, rather than immediately moving to a risk-neutral valuation procedure, we first present a simple Actuarial valuation approach for pricing exchange options in Section 2.2. This approach has been adopted by some industry participants and is justified by assuming that the risks associated with the energy prices are non-diversifiable (see for example Hull (2005)). Margrabe (1978) first valued exchange options assuming asset prices are GBMs under the risk-neutral measure and by utilizing a measure change induced by treating one of the assets as numeraire. However, since commodities are not liquid, their spot prices cannot act as a numeraire. Nonetheless, we show that there is a closely related measure change which renders the valuation in closed form even under the Actuarial approach.

Although some industry participants adopt an Actuarial valuation procedure, risk-neutral approaches are still very popular. In Section 3, we specify a class of equivalent risk-neutral measures which maintains the structure of the real-world dynamics. This allows us to reuse much of the valuation technology developed in Section 2.2. Once again, we show that an equivalent measure provides closed form pricing equations for spread options.

Most energy commodities are adequately modeled by diffusive processes; however, electricity prices themselves contain sever jumps. Section 4 is devoted to a jump-diffusion generalization of our previous results appropriate for spark-spread options - exchange options in which a fuel commodity is exchanged for electricity. Using the affine structure of our two-factor model with jumps, we obtain the forward prices as a solution to a system of coupled ODEs which we explicitly solve. Furthermore, through measure changes and Fourier transform methods, à la Duffie, Pan, and Singleton (2000) and Carr and Madan (1999), we obtain closed form formula for the price of spark-spread options on forwards.

We complete the paper in Section 5 with a calibration procedure that fits the model to spot and forward prices simultaneously. Calibrating to both spot and forward prices allows us to further extract the market prices of risk implied by the data. We apply the calibration procedure to the NYMEX Light Sweet Crude Oil spot and futures data and report on the stability of the implied model parameters as well as on the implied market prices of risk. Interestingly, the real-world mean-reversion rates are found to be higher than the risk-neutral mean-reversion rates. Furthermore, the rate of mean-reversion of the stochastic long-run mean level is lower than the mean-reversion rate of the log-spot price process. We find that these features are reflected in the market prices of risk themselves.

\section{Real World Dynamics and Pricing}

\subsection{Model Specifications}

A quick glance at historical spot prices for energy markets shows that traditional geometric Brownian motion models, even as a first order model, are inadequate. A successful model must include mean reversion as an essential feature. For early use of such models see the papers by Gibson and Schwartz (1990) and Cortazar and Schwartz (1994). These early one-factor models are a good first order model; however, as energy derivatives will often have maturities extending into months, or even years, such first 
order models require improvement. They invariably cannot match the term structure of forward prices for example. To this end, Pilipovic (1997) first suggested the two-factor mean-reverting model:

$d S_{t}=\beta\left(\theta_{t}-S_{t}\right) d t+\sigma_{S} S_{t} d W_{t}^{(1)}$,

$d \theta_{t}=\alpha \theta_{t} d t+\sigma_{\theta} \theta_{t} d W_{t}^{(2)}$,

where the two Brownian risk factors are correlated: $d\left[W^{(1)}, W^{(2)}\right]_{t}=\rho d t$. In this model, $\theta_{t}$ represents the stochastic long-run mean that spot prices $S_{t}$ revert to. This additional degree of stochasticity helps to correct some of the biases that a fixed long-run mean produces. In this parametrization, the mean reverting level is a geometric Brownian motion and can therefore grow without bound leading to nonstationary spot price processes. To circumvent this problem, and to assist with obtaining closed form formulae for spread options, we propose to model the log spot-price with an affine two-factor meanreverting process. Much like Pilipovic's model, the first factor mean-reverts to stochastic level; however, we ensure that the stochastic mean-reverting level also mean-reverts to a second long-run mean. With such a parametrization, the distribution of spot-prices is stationary, prices do not grow without bound, and the model remains within the Affine modeling class.

If the individual assets are driven by a two-factor model, then four driving factors are required to value spread options - two for each asset. Let $\left\{W_{t}^{(i)}\right\}_{0 \leq t \leq T}$ and $\left\{Z_{t}^{(i)}\right\}_{0 \leq t \leq T}$, with $i=1$ or 2 , denote these four Brownian risk factors and $\mathbb{F}=\left\{\mathcal{F}_{t}\right\}_{0 \leq t \leq T}$ denote the natural filtration generated by these processes. The measure $\mathbb{P}$ will denote the real-world probability distribution and $\{\Omega, \mathbb{F}, \mathbb{P}\}$ is used to denote the complete stochastic basis for the probability space. The spot-prices $\left\{S_{t}^{(i)}\right\}_{0 \leq t \leq T}$, with $i=1$ or 2 , are obtained via an exponentiation of the driving risk-factors. More specifically, the spot-prices are defined as follows:

$S_{t}^{(i)}:=\exp \left(g_{t}^{(i)}+X_{t}^{(i)}\right), \quad i=1,2$.

Seasonality is an important feature of some commodity prices; we therefore include the seasonality term $g_{t}^{(i)}$ using the following popular ansätz:

$g_{t}^{(i)}=A_{0}^{(i)} t+\sum_{k=1}^{n}\left(A_{k}^{(i)} \sin (2 \pi k t)+B_{k}^{(i)} \cos (2 \pi k t)\right)$.

For calibration stability, $n$ is typically kept small: $n=1$ or 2 . In our subsequent calculations we leave $g_{t}^{(i)}$ general, assuming only smoothness and differentiability.

To complete the specification of the two-factor model which drives the spot-prices, $X_{t}^{(i)}$ is assumed to satisfy the following coupled SDEs:

$d X_{t}^{(i)}=\beta_{i}\left(Y_{t}^{(i)}-X_{t}^{(i)}\right) d t+\sigma_{X}^{(i)} d W_{t}^{(i)}$,
$d Y_{t}^{(i)}=\alpha_{i}\left(\phi_{i}-Y_{t}^{(i)}\right) d t+\sigma_{Y}^{(i)} d Z_{t}^{(i)}$.

Here, $\beta_{i}$ controls the speed of mean-reversion of $X_{t}^{(i)}$ to the stochastic long-run level $Y_{t}^{(i)} ; \alpha_{i}$ controls the speed of mean-reversion of the long-run level $Y_{t}^{(i)}$ to the target long-run mean $\phi_{i} ; \sigma_{X}^{(i)}$ and $\sigma_{Y}^{(i)}$ control the size of the fluctuations around these means.

To reduce the parameter space, the measure $\mathbb{P}$ is chosen such that the following simple correlation structure is imposed on the Brownian motions:

$$
\begin{aligned}
d\left[W^{(1)}, W^{(2)}\right]_{t} & =\rho_{12} d t, \\
d\left[W^{(i)}, Z^{(i)}\right]_{t} & =\rho_{i} d t, \quad i=1,2,
\end{aligned}
$$

and all other cross correlations are zero. This structure allows the main driving factors $X_{t}^{(i)}$ to be correlated to one another and their own idiosyncratic long-run mean-reverting processes $Y_{t}^{(i)}$, while this structure forces the long-run reverting factors $Y_{t}^{(1)}$ and $Y_{t}^{(2)}$ to have an instantaneous correlation of zero. It is a straightforward, albeit tedious, matter to generalize this correlation structure. 
The coupled SDEs (6)-(7) can be solved by (i) first solving (7) for $Y_{t}^{(i)}-$ which is the standard meanreverting Ornstein-Uhlenbeck process - to obtain

$Y_{t}^{(i)}=\phi_{i}+\left(Y_{s}^{(i)}-\phi_{i}\right) e^{-\alpha_{i}(t-s)}+\sigma_{Y}^{(i)} \int_{s}^{t} e^{-\alpha_{i}(t-u)} d Z_{u}^{(i)} ;$

and then (ii) substituting this result into (6) to solve for $X_{t}^{(i)}$ while accounting for the correlation and feedback of $Y_{t}^{(i)}$ into $X_{t}^{(i)}$. After some tedious calculations $X_{t}^{(i)}$ can be represented as

$X_{t}^{(i)}=G_{s, t}^{(i)}+e^{-\beta_{i}(t-s)} X_{s}^{(i)}+M_{s, t}^{(i)} Y_{s}^{(i)}+\sigma_{X}^{(i)} \int_{s}^{t} e^{-\beta_{i}(t-u)} d W_{u}^{(i)}+\sigma_{Y}^{(i)} \int_{s}^{t} M_{u, t}^{(i)} d Z_{u}^{(i)}$,

where $\gamma_{i}:=\frac{\beta_{i}}{\alpha_{i}-\beta_{i}}$, and $G_{s, t}^{(i)}$ and $M_{s, t}^{(i)}$ are the deterministic functions

$$
\begin{aligned}
M_{s, t}^{(i)} & :=\gamma_{i}\left(e^{-\beta_{i}(t-s)}-e^{-\alpha_{i}(t-s)}\right), \\
G_{s, t}^{(i)} & :=\phi_{i}\left(1-e^{-\beta_{i}(t-s)}\right)-\phi_{i} M_{s, t}^{(i)} .
\end{aligned}
$$

Armed with our two-factor model and the solutions (10)-(11), we now focus our attention on the valuation of spot spread options and defer the valuation of spreads on forwards and model calibration to sections 3.4 and 5 respectively.

\subsection{Spot Spread Valuation : An Actuarial Approach}

Much like the financial markets, energy markets are rife with derivative products. However, one product stands out among the many that are traded over the counter: spread options. These options provide the owner with the right to exchange a specified quantity of one asset for another, at a fixed cost. The holder of such an option receives a maturity payoff of

$\varphi\left(S_{T}^{(1)}, S_{T}^{(2)}\right):=\max \left(S_{T}^{(1)}-\alpha S_{T}^{(2)}-K, 0\right)$.

When the cost of exchanging $K$ is set to zero, the option is known as a Margrabe or exchange option (Margrabe, 1978). Various approximations for the general (spot) spread option, under simple diffusion processes, have been studied in the literature and the reader is referred to Carmona and Durrleman (2003) for an excellent overview and further references. In the context of electricity markets, this option is known as the spark-spread option and $\alpha$ represents the heat rate of a given plant. The heat rate encapsulates the plant's profitability by specifying the number of units of the underlying commodity (such as oil or natural gas) which produces one unit of power - this product is studied in Section 4. If the exchange is between crude oil and a refined product (such as gasoline) the option is known as a crack-spread option. Many other specific examples of exchange options exist in the energy sector. In all cases, the exchange option can be used to hedge against market movements of spot prices or, alternatively, to speculate on those moves. In either case, a valuation framework is required.

It is difficult and sometimes not viable to store electricity and energy commodities; this results in an illiquid spot market. Harrison and Pliska (1981) demonstrated that the absence of arbitrage is equivalent to the existence of a measure, not necessarily unique, under which the relative price process of tradable assets to the money market account are martingales. Such measures are known as a risk-neutral measures. However, this conclusion has one important assumption - unrestricted and liquid trading of the underlying asset. In illiquid (spot) energy markets, it may be dubious to adopt a risk-neutral pricing framework, and although we ultimately proceed with that program, we first take an Actuarial approach. By assuming that the risks associated with the energy prices are non-diversifiable, it is possible to justify an Actuarial approach to pricing derivatives (Hull, 2005) which values an option as its discounted real-world expected payoff.

Definition. The Actuarial valuation principle assigns the following price to a T-maturity contingent claim with payoff $\varphi\left(S_{T}^{(1)}, S_{T}^{(2)}\right)$ :

$\Pi_{t, T}:=P(t, T) \mathbb{E}_{t}^{\mathbb{P}}\left[\varphi\left(S_{T}^{(1)}, S_{T}^{(2)}\right)\right]$. 
The notation $\mathbb{E}_{t}[\mathcal{A}]$ represents the expectation of $\mathcal{A}$ conditional on the filtration $\mathcal{F}_{t}$.

Throughout the article we assume the (possibly random) interest rates to be independent from other risk factors and write the price at time $t$ of a zero-coupon bond maturing at $T$ as $P(t, T)$.

In the traditional valuation procedure, expectations are taken under the risk-neutral measure $\mathbb{Q}$; here, however, the relevant measure is the real-world one $\mathbb{P}$. This complicates the problem somewhat. When the asset and the derivative are tradable, it is possible to use a numeraire change to value the Margrabe option; in the present context the asset cannot be used as numeraire and the relevant measure is not the risk-neutral one. Nonetheless, it is possible to adopt a similar strategy; to this end, define the auxiliary process

$H_{t, T}^{(i)}:=\mathbb{E}_{t}^{\mathbb{P}}\left[S_{T}^{(i)}\right]$.

If the expectation in (16) is computed under a risk-neutral measure, then $H_{t, T}^{(i)}$ represents the $T$-maturity forward price, which motivates us to coin $H_{t, T}^{(i)}$ the $T$-maturity pseudo-forward price process. At maturity this "price" process coincides with the spot-price $H_{T, T}^{(i)}=S_{T}^{(i)}$ allowing the Actuarial value of the exchange option to be written:

$\Pi_{t, T}=P(t, T) \mathbb{E}_{t}^{\mathbb{P}}\left[\left(H_{T, T}^{(1)}-\alpha H_{T, T}^{(2)}\right)_{+}\right]$.

The pseudo-forward price process has two other notable properties: (i) its expectation is bounded at all finite times $\mathbb{E}^{\mathbb{P}}\left[\left|H_{t, T}^{(i)}\right|\right]=H_{0, T}^{(i)}<+\infty$ for all $t<T$, and (ii) it is a $\mathbb{P}$-martingale. These two properties allow a normalized version of $H_{t, T}^{(i)}$ to assist in transforming the measure $\mathbb{P}$ into a particularly convenient measure for pricing. This measure change can, in some sense, be interpreted as being induced by using the pseudo-forward price process as a numeraire asset. The following Theorem contains one of our main tools.

Theorem. 2.1 Let $\left\{\eta_{t}\right\}_{0 \leq t \leq T}$ denote the Radon-Nikodym process

$\eta_{t}:=\left(\frac{d \widetilde{\mathbb{P}}}{d \mathbb{P}}\right)_{t}:=\frac{H_{t, T}^{(2)}}{H_{0, T}^{(2)}}$.

Then, for any $\mathcal{A} \in \mathcal{F}_{T}$

$\widetilde{\mathbb{P}}(\mathcal{A})=\mathbb{E}^{\mathbb{P}}\left[\mathbb{I}(\mathcal{A}) \eta_{T}\right]$,

and in particular $\widetilde{W}_{t}^{i}$ and $\widetilde{Z}_{t}^{i}(i=1,2)$ defined by

$$
\begin{aligned}
\widetilde{W}_{t}^{(2)} & =W_{t}^{(2)}-\int_{0}^{t}\left[\sigma_{X}^{(2)} e^{-\beta_{2}(T-u)}+\rho_{2} \sigma_{Y}^{(2)} M_{u, T}^{(2)}\right] d u \\
\widetilde{Z}_{t}^{(2)} & =Z_{t}^{(2)}-\int_{0}^{t}\left[\sigma_{Y}^{(2)} M_{u, T}^{(2)}+\rho_{2} \sigma_{X}^{(2)} e^{-\beta_{2}(T-u)}\right] d u \\
\widetilde{W}_{t}^{(1)} & =W_{t}^{(1)}-\int_{0}^{t}\left[\rho_{12} \sigma_{X}^{(2)} e^{-\beta_{2}(T-u)}\right] d u \\
\widetilde{Z}_{t}^{(1)} & =Z_{t}^{(1)}-\int_{0}^{t}\left[\rho_{1} \rho_{12} \sigma_{X}^{(2)} e^{-\beta_{2}(T-u)}\right] d u
\end{aligned}
$$

are $\widetilde{\mathbb{P}}-$ Wiener processes with correlation structure

$$
\begin{aligned}
d\left[\widetilde{W}^{(1)}, \widetilde{W}^{(2)}\right]_{t} & =\rho_{12} d t, \\
d\left[\widetilde{W}^{(i)}, \widetilde{Z}^{(i)}\right]_{t} & =\rho_{i} d t, \quad i=1,2,
\end{aligned}
$$

and all other cross correlations zero. 
Proof. Given properties (i) and (ii) above and $\eta_{0}=1$, it is clear that $\eta_{t}$ is a Radon-Nikodym derivative process and equation (19) immediately follows. To demonstrate that $\widetilde{W}_{t}^{(i)}$ and $\widetilde{Z}_{t}^{(i)}$ are $\widetilde{\mathbb{P}}$-Wiener processes substitute (11) into $H_{t, T}^{(i)}$ and then compute the expectation explicitly as follows:

$$
\begin{aligned}
H_{t, T}^{(i)}= & \mathbb{E}_{t}^{\mathbb{P}}\left[\operatorname { e x p } \left\{g_{T}^{(i)}+G_{t, T}^{(i)}+e^{-\beta_{i}(T-t)} X_{t}^{(i)}+M_{t, T}^{(i)} Y_{t}^{(i)}\right.\right. \\
& \left.\left.+\sigma_{X}^{(i)} \int_{t}^{T} e^{-\beta_{i}(T-u)} d W_{u}^{(i)}+\sigma_{Y}^{(i)} \int_{t}^{T} M_{u, T}^{(i)} d Z_{u}^{(i)}\right\}\right] \\
= & \exp \left(g_{T}^{(i)}+G_{t, T}^{(i)}+R_{t, T}^{(i)}+e^{-\beta_{i}(T-t)} X_{t}^{(i)}+M_{t, T}^{(i)} Y_{t}^{(i)}\right)
\end{aligned}
$$

Here,

$$
\begin{aligned}
R_{t, T}^{(i)}:= & \frac{h\left(t, T ; 2 \beta_{i}\right)}{2}\left[\left(\sigma_{X}^{(i)}\right)^{2}+\left(\gamma_{i} \sigma_{Y}^{(i)}\right)^{2}+2 \rho_{i} \gamma_{i} \sigma_{X}^{(i)} \sigma_{Y}^{(i)}\right] \\
& -h\left(t, T ; \alpha_{i}+\beta_{i}\right)\left[\left(\gamma_{i} \sigma_{Y}^{(i)}\right)^{2}+\rho_{i} \gamma_{i} \sigma_{X}^{(i)} \sigma_{Y}^{(i)}\right]+\frac{h\left(t, T ; 2 \alpha_{i}\right)}{2}\left(\gamma_{i} \sigma_{Y}^{(i)}\right)^{2},
\end{aligned}
$$

with

$h(t, T ; a):=\left(1-e^{-a(T-t)}\right) / a$.

The "Girsanov kernel" can now be read off directly from (26) and the solution for $X_{t}^{(i)}$ and $Y_{t}^{(i)}$ given in (10)-(11). Through Girsanov's theorem we find that (20)-(23) are $\widetilde{\mathbb{P}}$-Wiener processes.

Corollary 2.2 The Actuarial valuation formula (15) can be transformed to

$\Pi_{t, T}:=P(t, T) \mathbb{E}_{t}^{\mathbb{P}}\left[\varphi\left(H_{T, T}^{(1)}, H_{T, T}^{(2)}\right)\right]=P(t, T) H_{t, T}^{(2)} \mathbb{E}_{t}^{\widetilde{\mathbb{P}}}\left[\frac{\varphi\left(H_{T, T}^{(1)}, H_{T, T}^{(2)}\right)}{H_{T, T}^{(2)}}\right]$.

Proof. Use $\eta_{t}$ to change the measure.

It now remains to compute the expectation appearing in (29) under the transformed measure $\widetilde{\mathbb{P}}$. Recall that a process $M_{t}$ is a $\widetilde{\mathbb{P}}$-martingale if and only if the process $M_{t}(d \widetilde{\mathbb{P}} / d \mathbb{P})_{t}$ is a $\mathbb{P}$-martingale. Consequently, both $H_{t, T}:=H_{t, T}^{(1)} / H_{t, T}^{(2)}$ and $(d \mathbb{P} / d \widetilde{\mathbb{P}})_{t}$ are $\widetilde{\mathbb{P}}$-martingales. This, together with Corollary 2.2, reduces the Actuarial price of the Margrabe spread option to

$\Pi_{t, T}=P(t, T) H_{t, T}^{(2)} \mathbb{E}_{t}^{\widetilde{\mathbb{P}}}\left[\left(H_{T, T}-\alpha\right)_{+}\right]$

Since $H_{t, T}$ is a $\widetilde{\mathbb{P}}$-martingale, its drift under the $\widetilde{\mathbb{P}}$-measure is zero. Putting this together with equation (26), we find that $H_{t, T}$ satisfies the SDE:

$\frac{d H_{t, T}}{H_{t, T}}=\sigma_{X}^{(1)} e^{-\beta_{1}(T-t)} d \widetilde{W}_{t}^{(1)}-\sigma_{X}^{(2)} e^{-\beta_{2}(T-t)} d \widetilde{W}_{t}^{(2)}+\sigma_{Y}^{(1)} M_{t, T}^{(1)} d \widetilde{Z}_{t}^{(1)}-\sigma_{Y}^{(2)} M_{t, T}^{(2)} d \widetilde{Z}_{t}^{(2)}$

This expression clearly shows that $H_{t, T}$ is a geometric Brownian motion with time dependent (but deterministic) volatility; consequently, its terminal value can be expressed in terms of its initial value via $H_{T, T}=H_{t, T} \exp \left(U_{t, T}\right)$, where $U_{t, T}$ is a normal random variable with mean equal to $-\frac{1}{2}\left(\sigma_{t, T}\right)^{2}$ and variance equal to $\left(\sigma_{t, T}\right)^{2}$. Here,

$$
\begin{gathered}
\left(\sigma_{t, T}\right)^{2}=\mathbb{E}_{t}^{\widetilde{\mathbb{P}}}\left[\left(\sigma_{X}^{(1)} \int_{t}^{T} e^{-\beta_{1}(T-s)} d \widetilde{W}_{s}^{(1)}-\sigma_{X}^{(2)} \int_{t}^{T} e^{-\beta_{2}(T-s)} d \widetilde{W}_{s}^{(2)}\right.\right. \\
\left.\left.+\sigma_{Y}^{(1)} \int_{t}^{T} M_{s, T}^{(1)} d \widetilde{Z}_{s}^{(1)}-\sigma_{Y}^{(2)} \int_{t}^{T} M_{s, T}^{(2)} d \widetilde{Z}_{s}^{(2)}\right)^{2}\right] \\
=2 R_{t, T}^{(1)}+2 R_{t, T}^{(2)}-2 \rho_{12} \sigma_{X}^{(1)} \sigma_{X}^{(2)} h\left(t, T ; \beta_{1}+\beta_{2}\right) .
\end{gathered}
$$


The deterministic functions $M_{s, T}^{(i)}$ and $R_{t, T}^{(i)}$ are as in (12) and (27) respectively. It is now a straightforward matter to recover the final result of this section - a "Black-Scholes like" expression for the Actuarial price of the exchange option.

Proposition 2.3 The Actuarial value at time $t$ of the T-maturity exchange option is

$\Pi_{t, T}=P(t, T)\left[H_{t, T}^{(1)} \Phi\left(d+\sigma_{t, T}\right)-\alpha \cdot H_{t, T}^{(2)} \Phi(d)\right]$

with $\sigma_{t, T}$ as in (32) and $d$ defined as

$d:=\frac{\log \frac{H_{t, T}}{\alpha}-\frac{1}{2}\left(\sigma_{t, T}\right)^{2}}{\sigma_{t, T}}$.

\section{Risk-Neutral Dynamics and Pricing}

In complete market settings, there exists a unique equivalent measure which induces the relative price process of tradable assets to be martingales. This measure is known as the risk-neutral measure $\mathbb{Q}$. In the present context, the underlying asset is not tradable in the usual sense due to the illiquidity issue and potentially large storage costs. In the previous section we dealt with this issue by resorting to an Actuarial valuation procedure and assigned a price equal to the discounted expectation of the terminal payoff under the real-world measure. However, one can in principle still utilize risk-neutral methodologies adjusting for the incompleteness of the market settings. Within such incomplete markets there may exists many equivalent risk-neutral measures; it is the job of the market as a whole, via trading of derivatives, to decide which measure prevails at any one given point in time. In this next section, we provide a class of equivalent martingale measures that maintains the structure of our real-world dynamics for asset prices. These measures are then used to obtain forward prices and value spread options.

\subsection{Measure Change}

In this section, we introduce a class of risk-neutral measure changes which maintains the real-world structure of the asset dynamics. The following Lemma introduces the new measure induced by a four dimensional market price of risk vector.

Lemma 3.1 Let $\left\{\zeta_{t}\right\}_{0 \leq t \leq T}$ denote the Radon-Nikodym process,

$\zeta_{t}:=\left(\frac{d \mathbb{Q}}{d \mathbb{P}}\right)_{t}=\mathcal{E}\left(\int_{0}^{t}\left\{\lambda_{u}^{(1)} d W_{u}^{(1)}+\psi_{u}^{(1)} d Z_{u}^{(1)}+\lambda_{u}^{(2)} d W_{u}^{(2)}+\psi_{u}^{(2)} d Z_{u}^{(2)}\right\}\right)$,

where $\mathcal{E}\left(\mathcal{A}_{t}\right)$ is the Dolean-Dade's exponential of the process $\mathcal{A}_{t}$. Then for any $\mathcal{A} \in \mathcal{F}_{T}$ we have,

$\mathbb{Q}(\mathcal{A})=\mathbb{E}^{\mathbb{P}}\left[\mathcal{A} \zeta_{T}\right]$.

In particular the following are $\mathbb{Q}$-Wiener processes:

$$
\begin{aligned}
& \bar{W}_{t}^{(1)}=W_{t}^{(1)}-\int_{0}^{t}\left\{\lambda_{u}^{(1)}+\rho_{12} \lambda_{u}^{(2)}+\rho_{1} \psi_{u}^{(1)}\right\} d u \\
& \bar{Z}_{t}^{(1)}=Z_{t}^{(1)}-\int_{0}^{t}\left\{\rho_{1} \lambda_{u}^{(1)}+\psi_{u}^{(1)}\right\} d u \\
& \bar{W}_{t}^{(2)}=W_{t}^{(2)}-\int_{0}^{t}\left\{\rho_{12} \lambda_{u}^{(1)}+\lambda_{u}^{(2)}+\rho_{2} \psi_{u}^{(2)}\right\} d u \\
& \bar{Z}_{t}^{(2)}=Z_{t}^{(2)}-\int_{0}^{t}\left\{\rho_{2} \lambda_{u}^{(2)}+\psi_{u}^{(2)}\right\} d u
\end{aligned}
$$

with correlation structure,

$$
\begin{aligned}
d\left[\bar{W}^{(1)}, \bar{W}^{(2)}\right]_{t} & =\rho_{12} d t \\
d\left[\bar{W}^{(i)}, \bar{Z}^{(i)}\right]_{t} & =\rho_{i} d t, \quad i=1,2,
\end{aligned}
$$


and all other cross-correlations zero.

Proof. Decompose the correlated processes into uncorrelated processes and apply Girsanov's Theorem.

Notice that there are no restrictions on the form of the market prices of risk other than the usual integrability ones. In particular, the drifts under the risk-neutral measure $\mathbb{Q}$ are not constrained to the risk-free rate. This is precisely the effect of incompleteness in the present context. The following Theorem applies constraints on the market prices of risk such that the risk-neutral dynamics and the real-world one are of the same form.

Theorem. 3.2 If the market price of risk processes are chosen as follows:

$\lambda_{t}^{(i)}=\lambda^{(i)}+\lambda_{X}^{(i)} X_{t}^{(i)}+\lambda_{Y}^{(i)} Y_{t}^{(i)}$,

$\psi_{t}^{(i)}=\psi^{(i)}+\psi_{X}^{(i)} X_{t}^{(i)}+\psi_{Y}^{(i)} Y_{t}^{(i)}$,

subject to the constraints $((i, j) \in\{(1,2),(2,1)\})$

$$
\begin{aligned}
\psi_{X}^{(i)} & =-\rho_{i} \lambda_{X}^{(i)}, \\
\lambda^{(i)}+\rho_{12} \lambda^{(j)}+\rho_{i} \psi^{(i)} & =0 \\
\lambda_{X}^{(i)}+\rho_{12} \lambda_{X}^{(j)}+\rho_{i} \psi_{X}^{(i)} & =-\left(\lambda_{Y}^{(i)}+\rho_{12} \lambda_{Y}^{(j)}+\rho_{i} \psi_{Y}^{(i)}\right), \\
\bar{\alpha}_{i} & =\alpha_{i}-\sigma_{Y}^{(i)}\left(\rho_{i} \lambda_{Y}^{(i)}+\psi_{Y}^{(i)}\right), \\
\bar{\alpha}_{i} \bar{\phi}_{i} & =\left(\alpha_{i} \phi_{i}+\sigma_{Y}^{(i)}\left(\rho_{i} \lambda^{(i)}+\psi^{(i)}\right)\right), \\
\bar{\beta}_{i} & =\beta_{i}+\sigma_{X}^{(i)}\left(\lambda_{Y}^{(i)}+\rho_{12} \lambda_{Y}^{(j)}+\rho_{i} \psi_{Y}^{(i)}\right),
\end{aligned}
$$

then the risk-neutral dynamics of $X_{t}^{(i)}$ and $Y_{t}^{(i)}$ remain within the same class as (6)-(7). In particular,

$d X_{t}^{(i)}=\bar{\beta}_{i}\left(Y_{t}^{(i)}-X_{t}^{(i)}\right) d t+\sigma_{X}^{(i)} d \bar{W}_{t}^{(i)}$

$d Y_{t}^{(i)}=\bar{\alpha}_{i}\left(\bar{\phi}_{i}-Y_{t}^{(i)}\right) d t+\sigma_{Y}^{(i)} d \bar{Z}_{t}^{(i)}$.

Proof. Insert the expressions for the $\mathbb{Q}$-Wiener processes $\bar{W}_{t}^{(i)}$ and $\bar{Z}_{t}^{(i)}$ into (6)-(7). Collect similar terms and equate coefficients.

This ansätz may seem restrictive; however, even though the risk-neutral dynamics remains within the same class as the real-world one, the coefficients driving that dynamics may be significantly different. This flexibility is sufficient for the simultaneous calibration of the risk-neutral and real-world model parameters, while remaining parsimonious.

\subsection{Forward Prices}

Since the risk-neutral dynamics of the driving diffusion processes (51)-(52) are of the same form as they were under the objective measure (6)-(7), the forward price curves can easily be extracted from equation (26). This is because, within a risk-neutral framework, the forward prices are defined as

$F_{t, T}^{(i)}:=\mathbb{E}_{t}^{\mathbb{Q}}\left[S_{T}^{(i)}\right]$,

the precise risk-neutral analog of the pseudo-forward price defined in (16). All that remains is to change the $\mathbb{P}$-parameters for the $\mathbb{Q}$-parameters. 
Proposition 3.3 The forward prices associated to commodity $i=1,2$ are given by

$F^{(i)}(t, T)=\exp \left(g_{T}^{(i)}+\bar{R}_{t, T}^{(i)}+\bar{G}_{t, T}^{(i)}+e^{-\bar{\beta}_{i}(T-t)} X_{t}^{(i)}+\bar{M}_{t, T}^{(i)} Y_{t}^{(i)}\right)$

where the expressions for $\bar{M}_{t, T}^{(i)}, \bar{G}_{t, T}^{(i)}$ and $\bar{R}_{t, T}^{(i)}$ are supplied in equations (12)-(13) and (27) respectively - with the appropriate change of parameters $\left(\alpha_{i} \rightarrow \bar{\alpha}_{i}\right.$ and so on...).

These results can be viewed as an extension of the one-factor model Cartea and Figueroa (2005) study, albeit without jumps. In section 4 , we address the two factor model with jumps.

\subsection{Spot Spread Valuation}

To value the option under a risk-neutral measure, we follow along the same lines as in Section 2.2. In the present context, the measure change is the one induced by using the forward price to drive the measure change. To this end, define a new measure $\widetilde{\mathbb{Q}}$ via the Radon-Nikodym derivative process

$$
\left(\frac{d \widetilde{\mathbb{Q}}}{d \mathbb{Q}}\right)_{t}=\frac{\mathbb{E}_{t}^{\mathbb{Q}}\left[S_{T}^{(2)}\right]}{\mathbb{E}_{0}^{\mathbb{Q}}\left[S_{T}^{(2)}\right]}=\frac{F_{t, T}^{(2)}}{F_{0, T}^{(2)}}
$$

All steps leading to Proposition 2.3 remain valid in this new context, and rather than repeating them, we simply quote our final risk-neutral pricing result.

Proposition 3.4 The risk neutral value at time $t$ of the exchange option with maturity $T$ is:

$\Pi_{t, T}=P(t, T)\left[F_{t, T}^{(1)} \Phi\left(d+\sigma_{t, T}\right)-\alpha F_{t, T}^{(2)} \Phi(d)\right]$

where $d$ defined as

$d:=\frac{\log \frac{F_{t, T}}{\alpha}-\frac{\left(\sigma_{t, T}\right)^{2}}{2}}{\sigma_{t, T}}$

and $\sigma_{t, T}$ as in (32) - with all $\mathbb{P}$-parameters replaced by $\mathbb{Q}$-parameters (i.e., $\alpha_{i} \rightarrow \bar{\alpha}_{i}$ and so on...).

It is important to note that the market provides quotes for the forward curve $F_{t, T}^{(i)}, i=1,2$ for a set of maturities $T=\left\{T_{1}, \ldots, T_{n}\right\}$. These curves can be used to calibrate the risk-neutral parameters. Once the model has been calibrated to market data, the resulting pricing rules are just as simple to use as the Black-Scholes formula for a European option on a single asset. Although the explicit expressions for the forward prices $F_{t, T}^{(i)}$ and the effective volatility $\sigma_{t, T}$ are somewhat bulky, they involve nothing more complex than exponentiation and are therefore very efficient to calculate.

\subsection{Forward Spread Valuation}

In the previous sections we focused on valuing a spread option based on the future spot prices; however, a more popular derivative product involves the spread between the forward prices of the two assets (possibly with differing maturities). Such spreads on forwards pay

$\varphi\left(F_{T, T_{1}}^{(1)}, F_{T, T_{2}}^{(2)}\right)=\left(F_{T, T_{1}}^{(1)}-\alpha F_{T, T_{2}}^{(2)}\right)_{+}$

at the maturity date $T$ where it is implicit that $T_{1}, T_{2} \geq T$. We can once again use a measure change to simplify the calculations, this time it is convenient to use the $T_{2}$-maturity forward price of asset 2, i.e. $F_{t, T_{2}}^{(2)}$, to induce a measure change. In particular we define the Radon-Nikodym derivative process

$\left(\frac{d \widetilde{\mathbb{Q}}}{d \mathbb{Q}}\right)_{t}:=\frac{F_{t, T_{2}}^{(2)}}{F_{0, T_{2}}^{(2)}}$.

The time $t$ price of the forward spread option is therefore

$\Pi_{t, T}^{F}:=P(t, T) \mathbb{E}_{t}^{\mathbb{Q}}\left[\left(F_{T, T_{1}}^{(1)}-\alpha F_{T, T_{2}}^{(2)}\right)_{+}\right]=P(t, T) F_{t, T_{2}}^{(2)} \mathbb{E}_{t}^{\widetilde{\mathbb{Q}}}\left[\left(F_{T ; T_{1}, T_{2}}-\alpha\right)_{+}\right]$. 
Here, $F_{t ; T_{1}, T_{2}}:=F_{t, T_{1}}^{(1)} / F_{t, T_{2}}^{(2)}$ is the ratio of the two relevant forward prices. In analogy with our earlier calculations, the relative process $F_{t ; T_{1}, T_{2}}$ is a $\widetilde{\mathbb{Q}}$-martingale and therefore its $\widetilde{\mathbb{Q}}$-dynamics is driftless. Following along the same arguments as in Section 2.2, it is easy to show that $F_{T ; T_{1}, T_{2}}=F_{t ; T_{1}, T_{2}} \exp \left\{U_{t ; T, T_{1}, T_{2}}^{*}\right\}$ where $U_{t ; T, T_{1}, T_{2}}^{*}$ is a normal random variable with mean equal to $-\frac{1}{2}\left(\sigma_{t ; T, T_{1}, T_{2}}^{*}\right)^{2}$ and variance equal to $\left(\sigma_{t ; T, T_{1}, T_{2}}^{*}\right)^{2}$. The explicit form for the variance is

$$
\begin{aligned}
\left(\sigma_{t ; T, T_{1}, T_{2}}^{*}\right)^{2}:= & \left(\bar{\gamma}_{1} \sigma_{Y}^{(1)}\right)^{2}\left[h\left(t, T_{1} ; 2 \bar{\alpha}_{1}\right)-h\left(T, T_{1} ; 2 \bar{\alpha}_{1}\right)\right] \\
& +\left(\bar{\gamma}_{2} \sigma_{Y}^{(2)}\right)^{2}\left[h\left(t, T_{2} ; 2 \bar{\alpha}_{2}\right)-h\left(T, T_{2} ; 2 \bar{\alpha}_{2}\right)\right] \\
& +\left\{\left(\sigma_{X}^{(1)}\right)^{2}+\left(\bar{\gamma}_{1} \sigma_{Y}^{(1)}\right)^{2}+2 \rho_{1} \bar{\gamma}_{1} \sigma_{X}^{(1)} \sigma_{Y}^{(1)}\right\}\left[h\left(t, T_{1} ; 2 \bar{\beta}_{1}\right)-h\left(T, T_{1} ; 2 \bar{\beta}_{1}\right)\right] \\
& +\left\{\left(\sigma_{X}^{(2)}\right)^{2}+\left(\bar{\gamma}_{2} \sigma_{Y}^{(2)}\right)^{2}+2 \rho_{2} \bar{\gamma}_{2} \sigma_{X}^{(2)} \sigma_{Y}^{(2)}\right\}\left[h\left(t, T_{2} ; 2 \bar{\beta}_{2}\right)-h\left(T, T_{2} ; 2 \bar{\beta}_{2}\right)\right] \\
& -\left\{2\left(\bar{\gamma}_{1} \sigma_{Y}^{(1)}\right)^{2}+2 \rho_{1} \bar{\gamma}_{1} \sigma_{X}^{(1)} \sigma_{Y}^{(1)}\right\}\left[h\left(t, T_{1} ; \bar{\alpha}_{1}+\bar{\beta}_{1}\right)-h\left(T, T_{1} ; \bar{\alpha}_{1}+\bar{\beta}_{1}\right)\right] \\
& -\left\{2\left(\bar{\gamma}_{2} \sigma_{Y}^{(2)}\right)^{2}+2 \rho_{2} \bar{\gamma}_{2} \sigma_{X}^{(2)} \sigma_{Y}^{(2)}\right\}\left[h\left(t, T_{2} ; \bar{\alpha}_{2}+\bar{\beta}_{2}\right)-h\left(T, T_{2} ; \bar{\alpha}_{2}+\bar{\beta}_{2}\right)\right] \\
& -2 \rho_{12} \sigma_{X}^{(1)} \sigma_{X}^{(2)} \exp \left\{-\bar{\beta}_{1}\left(T_{1}-T\right)-\bar{\beta}_{2}\left(T_{2}-T\right)\right\} h\left(t, T ; \bar{\beta}_{1}+\bar{\beta}_{2}\right)
\end{aligned}
$$

where, $h(t, T ; a)$ is defined in (28). The pricing equation (60) now reduces to a Black-Scholes like pricing result.

Proposition 3.5 The risk neutral value at time $t$ of the forward spread option (58) is

$\Pi_{t, T}^{F}=P(t, T)\left[F_{t, T_{1}}^{(1)} \Phi\left(d^{*}+\sigma_{t, T}^{*}\right)-\alpha F_{t, T_{2}}^{(2)} \Phi\left(d^{*}\right)\right]$

with $\left(\sigma_{t, T}^{*}\right)^{2}$ as in (61) and $d^{*}$ defined as

$d^{*}:=\frac{\log \frac{F_{t ; T_{1}, T_{2}}}{\alpha}-\frac{1}{2}\left(\sigma_{t, T}^{*}\right)^{2}}{\sigma_{t, T}^{*}}$.

Not surprisingly, the pricing result is very similar to the one in Proposition 3.4 and reduces to it when $T=T_{1}=T_{2}$.

\section{Spot Prices with Jumps}

The two factor diffusion model captures the main characteristics of most energy spot prices, however, it cannot account for the possibility of sudden jumps in the price data. Such behavior is particularly important for modeling electricity prices and various spreads contingent on electricity and other hedging assets. The most important example of such option is the so-called spark-spread option which pays $\left(F_{T, T_{1}}^{(1)}-\alpha F_{T, T_{2}}^{(2)}-K\right)_{+}$at the maturity date $T$. Here $F_{t, T_{1}}^{(1)}:=\mathbb{E}_{t}^{\mathbb{Q}}\left[S_{T_{1}}^{(1)}\right]$ represents the electricity forward price, $F_{t, T_{2}}^{(2)}$ represents the forward price of the commodity used to generate electricity, and $\alpha$ represents the heat rate which encapsulates the number of units of energy that the plant produces per unit of commodity. Notice that the structure of this option allows forward prices of differing maturities to be used as the underlying. As commented earlier on, closed form solutions, even for the purely diffusion case, are not accessible for general strike levels; consequently, we limit ourselves to the exchange option with a strike of zero.

\subsection{Model Specification}

For brevity, we now focus solely on the risk-neutral valuation procedure, and provide model specifications directly under the risk-neutral measure. Typically, when electricity prices jump they revert 
back to normal levels very quickly. A widely used model specification incorporates jumps and diffusions simultaneously as follows:

$d \ln \left(S_{t}\right)=\alpha\left(\theta-\ln \left(S_{t-}\right)\right) d t+\sigma d W_{t}+d Q_{t}$.

Regardless of the specification of the jump process $Q_{t}$, such models suffer from unrealistically large diffusive volatilities and mean-reversion rates. This occurs because the process must revert very quickly to normal levels after a large jump, implying a high mean-reversion rate $\alpha$. This in turn induces an artificially large diffusive volatility, since otherwise all diffusive components would revert to the mean extremely quickly and, excluding the jumps, the paths would appear essentially deterministic.

We avoid these problems by splitting the jump component from the diffusion component and modeling them separately. Specifically, define the (power) spot price by

$S_{t}^{(1)}:=\exp \left\{g_{t}^{(1)}+X_{t}^{(1)}+J_{t}\right\}$,

where $X_{t}^{(i)}$ and $Y_{t}^{(i)}$ satisfy the usual two-factor SDEs (51)-(52), and the new jump component $J_{t}$ is defined via

$d J_{t}=-\kappa J_{t-} d t+d Q_{t}$,

with $Q_{t}$ a compound Poisson process: $Q_{t}:=\sum_{1}^{N_{t}} l_{i}$, where $N_{t}$ is a time inhomogeneous Poisson process with activity rate $\lambda(s)$, and $\left\{l_{i}\right\}$ the set of i.i.d. jump sizes with distribution function $F_{l}(u)$. Furthermore, $J_{t-}$ denotes the value of $J_{t}$ prior to any jump at time $t$. The jump component $J_{t}$ mean-reverts to zero with rate $\kappa$; typically, $\kappa$ will be quite large because electricity prices revert back to normal very quickly after a jump. This empirical fact has no direct bearing on the valuation procedure, however, it does attribute to the manner in which we have split the jump component from the diffusion component. We allow the activity rate to vary with time to permit seasonality effects in the rate of jump arrivals; however, we restrict it to be deterministic - it is possible to generalize to stochastic activity rates; however, the additional modeling flexibility renders the calibration process unstable. Finally, it is well known that diffusions and jump processes cannot have any instantaneous correlations, while this does not preclude the jump size from depending on the Brownian risk factors we make the natural assumption that $N_{t}$ and $\left\{l_{i}\right\}$ are independent of all the $\mathbb{Q}$-Brownian processes.

\subsection{Forward Prices}

Equipped with this jump-diffusion model, we now derive the forward price $F_{t, T}^{(1)}$ associated with the spot $S_{t}^{(1)}$. As usual, the forward price is the risk-neutral expectation of the asset price at the maturity

$f\left(t, X_{t}^{(1)}, Y_{t}^{(1)}, J_{t}\right):=F_{t, T}^{(1)}:=\mathbb{E}_{t}^{\mathbb{Q}}\left[S_{T}^{(1)}\right]=\mathbb{E}_{t}^{\mathbb{Q}}\left[\exp \left\{g_{T}^{(1)}+X_{T}^{(1)}+J_{T}\right\}\right]$.

Rather than computing this expectation directly, we make use of the affine form of the processes along the lines of Duffie, Pan, and Singleton (2000). Since $f$ is a $\mathbb{Q}$-martingale, it satisfies the zero-drift condition $\mathcal{A} f=0$ where $\mathcal{A}$ is the generator of the process $\left(t, X_{t}^{(1)}, Y_{t}^{(1)}, J_{t}\right)$. The affine ansätz:

$f\left(t, X_{t}^{(1)}, Y_{t}^{(1)}, J_{t}\right)=\exp \left\{A(t, T)+B(t, T) X_{t}^{(1)}+C(t, T) Y_{t}^{(1)}+D(t, T) J_{t}\right\}$,

with terminal conditions $A(T, T)=g_{T}^{(1)}, B(T, T)=1, C(T, T)=0$ and $D(T, T)=1$, reduces the PDE $\mathcal{A} f=0$ to the equivalent system of coupled ODEs:

$$
\begin{aligned}
B_{t}-\bar{\beta}_{1} B & =0, \\
C_{t}+\bar{\beta}_{1} B-\bar{\alpha}_{1} C & =0, \\
D_{t}-\kappa D & =0, \\
A_{t}+\bar{\alpha}_{1} \bar{\phi}_{1} C+\frac{\left(\sigma_{X}^{(1)}\right)^{2}}{2} B^{2}+\frac{\left(\sigma_{Y}^{(1)}\right)^{2}}{2} C^{2}+\rho_{1} \sigma_{X}^{(1)} \sigma_{Y}^{(1)} B C & =-\int_{-\infty}^{\infty} \lambda(u)\left(e^{D \cdot u}-1\right) d F_{l}(u) .
\end{aligned}
$$

Although rather tedious, standard methods can be used to solve this system and obtain the forward price. 
Proposition 4.1 The forward price for the two-factor jump-diffusion spot process $S_{t}^{(1)}$ is

$F_{t, T}^{(1)}=\exp \left\{A_{t, T}^{(1)}+e^{-\bar{\beta}_{1}(T-t)} X_{t}^{(1)}+\bar{M}_{t, T}^{(1)} Y_{t}^{(1)}+e^{-\kappa(T-t)} J_{t}\right\}$

where the deterministic function $\bar{M}_{t, T}^{(1)}$ is provided in equation (12),

$$
\begin{aligned}
A_{t, T}^{(1)}= & g_{T}^{(1)}+\int_{t}^{T} \lambda(s)\left(\varphi_{l}\left(e^{-\kappa(T-s)}\right)-1\right) d s \\
& -\bar{\alpha}_{1} \bar{\gamma}_{1} \bar{\phi}_{1}\left[h\left(t, T ; \bar{\alpha}_{1}\right)-h\left(t, T ; \bar{\beta}_{1}\right)\right] \\
& +\frac{1}{2}\left(\bar{\gamma}_{1} \sigma_{Y}^{(1)}\right)^{2}\left[h\left(t, T ; 2 \bar{\alpha}_{1}\right)+h\left(t, T ; 2 \bar{\beta}_{1}\right)-2 h\left(t, T ; \bar{\alpha}_{1}+\bar{\beta}_{1}\right)\right] \\
& +\frac{1}{2}\left(\sigma_{X}^{(1)}\right)^{2} h\left(t, T ; 2 \bar{\beta}_{1}\right)+\rho_{1} \bar{\gamma}_{1} \sigma_{X}^{(1)} \sigma_{Y}^{(1)}\left[h\left(t, T ; 2 \bar{\beta}_{1}\right)-h\left(t, T ; \bar{\alpha}_{1}+\bar{\beta}_{1}\right)\right],
\end{aligned}
$$

and $\varphi_{l}(u)$ is the m.g.f. of the individual jump sizes $l_{i}$,

$\varphi_{l}(u):=\mathbb{E}^{\mathbb{Q}}\left[e^{u l_{1}}\right]=\int_{-\infty}^{\infty} e^{u z} d F_{l}(z)$.

These results can be viewed as an extension of the one-factor model Cartea and Figueroa (2005) study.

\subsection{Spark Spread Valuation}

We now turn to the pricing of the (exchange) spark spread option with $T$-terminal payoff $\left(F_{T, T_{1}}^{(1)}-\alpha F_{T, T_{2}}^{(2)}\right)_{+}$ and $T \leq T_{1}, T_{2}$. As usual, the forward prices are expressed as $F_{t, T}^{(i)}:=\mathbb{E}_{t}^{\mathbb{Q}}\left[S_{T}^{(i)}\right]$ where $S_{t}^{(1)}:=$ $\exp \left\{g_{t}^{(1)}+X_{t}^{(1)}+J_{t}\right\}$ is the two-factor jump-diffusion spot price presented in the previous section and $S_{t}^{(2)}:=\exp \left\{g_{t}^{(2)}+X_{t}^{(2)}\right\}$ is the pure diffusion process of Section 3. We begin our analysis by rewriting the risk-neutral pricing formula in terms of an equivalent measure induced by the forward price process of the purely diffusive asset. In particular,

$\Pi_{t, T}^{F}:=P(t, T) \mathbb{E}_{t}^{\mathbb{Q}}\left[\left(F_{T, T_{1}}^{(1)}-\alpha F_{T, T_{2}}^{(2)}\right)_{+}\right]=P(t, T) F_{t, T_{2}}^{(2)} \mathbb{E}_{t}^{\widetilde{\mathbb{Q}}}\left[\left(F_{T ; T_{1}, T_{2}}-\alpha\right)_{+}\right]$,

where the measure $\widetilde{\mathbb{Q}}$ is induced by

$\left(\frac{d \widetilde{\mathbb{Q}}}{d \mathbb{Q}}\right)_{t}:=\frac{\mathbb{E}_{t}^{\mathbb{Q}}\left[S_{T_{2}}^{(2)}\right]}{\mathbb{E}_{0}^{\mathbb{Q}}\left[S_{T_{2}}^{(2)}\right]}=\frac{F_{t, T_{2}}^{(2)}}{F_{0, T_{2}}^{(2)}}$

and we introduced the ratio process $F_{t ; T_{1}, T_{2}}:=F_{t, T_{1}}^{(1)} / F_{t, T_{2}}^{(2)}$. The process $F_{t ; T_{1}, T_{2}}$ is once again a $\widetilde{\mathbb{Q}}-$ martingale; however, because of the presence of the jump component, this fact alone does not allow us to extract its distribution. Instead, we make use of transform methods. Carr and Madan (1999) were among the first to illustrate that Fast Fourier transform (FFT) methods can be used to efficiently value European options. The reader is referred to their work for implementation details and other efficiency tricks.

The FFT methods require the m.g.f. of the logarithm of the effective stochastic process - in our case the process $F_{t ; T_{1}, T_{2}}$. To this end, define $Z_{T}:=\ln F_{T ; T_{1}, T_{2}}$ so that

$$
\begin{aligned}
Z_{T}=A_{T, T_{1}}^{(1)} & -A_{T, T_{2}}^{(2)}+e^{-\bar{\beta}_{1}\left(T_{1}-T\right)} X_{T}^{(1)}-e^{-\bar{\beta}_{2}\left(T_{2}-T\right)} X_{T}^{(2)}+\bar{M}_{T, T_{1}}^{(1)} Y_{T}^{(1)} \\
& -\bar{M}_{T, T_{2}}^{(2)} Y_{T}^{(2)}+e^{-\kappa\left(T_{1}-T\right)} J_{T},
\end{aligned}
$$

and define the corresponding m.g.f. process

$\Psi_{t}^{Z_{T}}(u):=\mathbb{E}_{t}^{\widetilde{\mathbb{Q}}}\left[e^{u Z_{T}}\right]$ 
The process $\Psi_{t}^{Z_{T}}(u)$ is clearly a $\widetilde{\mathbb{Q}}$-martingale; consequently, it satisfies the zero drift condition $\mathcal{A} \Psi_{t}^{Z_{T}}(u)=$ 0 (for every $u$ where it is defined) where $\mathcal{A}$ is the generator of the process $\left(t, X_{t}^{(1)}, X_{t}^{(2)}, Y_{t}^{(1)}, Y_{t}^{(2)}, J_{t}\right)$ under $\widetilde{\mathbb{Q}}$. Furthermore, since our modeling framework is affine, we employ the ansätz

$$
\begin{aligned}
\Psi_{t}^{Z_{T}}(u):=\exp \{A(t, T)+ & B^{(1)}(t, T) X_{t}^{(1)}+B^{(2)}(t, T) X_{t}^{(2)}+C^{(1)}(t, T) Y_{t}^{(1)} \\
& \left.+C^{(2)}(t, T) Y_{t}^{(2)}+D(t, T) J_{t}\right\} \\
\Psi_{T}^{Z_{T}}(u):=\exp \left\{u Z_{T}\right\} &
\end{aligned}
$$

Here, $A(t, T), B^{(1)}(t, T), B^{(2)}(t, T), C^{(1)}(t, T), C^{(2)}(t, T)$, and $D(t, T)$ are all deterministic functions of time. Note that $T_{1}$ and $T_{2}$ have been removed from the arguments for easier readability. Since the boundary condition (81) must hold for all terminal values of the auxiliary processes $\left(X_{t}^{(1)}, X_{t}^{(2)}, Y_{t}^{(1)}, Y_{t}^{(2)}, J_{t}\right)$, the deterministic functions must satisfy the induced boundary conditions

$$
\begin{array}{ll}
A(T, T)=u\left(A_{T, T_{1}}^{(1)}-A_{T, T_{2}}^{(2)}\right), & B^{(1)}\left(T, T_{1}\right)=u e^{-\bar{\beta}_{1}\left(T_{1}-T\right)}, \\
B^{(2)}\left(T, T_{2}\right)=-u e^{-\bar{\beta}_{2}\left(T_{2}-T\right)}, & C^{(1)}\left(T, T_{1}\right)=u \bar{M}_{T, T_{1}}^{(1)} \\
C^{(2)}\left(T, T_{2}\right)=-u \bar{M}_{T, T_{2}}^{(2)}, & D\left(T, T_{1}\right)=u e^{-\kappa\left(T_{1}-T\right)} .
\end{array}
$$

Expanding the PDE $\mathcal{A} f=0$, rewriting it in terms of an equivalent system of coupled ODEs and solving that system (similar to the analysis in Section 4.2) provides the final result.

Proposition 4.2 The transform $\Psi_{t}^{Z_{T}}(u):=\mathbb{E}_{t}^{\widetilde{\mathbb{Q}}}\left[e^{u Z_{T}}\right]$ is given by

$$
\begin{array}{r}
\Psi_{t}^{Z_{T}}(u)=\exp \left\{A_{t, T}+u\left(e^{-\bar{\beta}_{1}\left(T_{1}-t\right)} X_{t}^{(1)}-e^{-\bar{\beta}_{2}\left(T_{2}-t\right)} X_{t}^{(2)}+\bar{M}_{t, T_{1}}^{(1)} Y_{t}^{(1)}\right.\right. \\
\left.\left.-\bar{M}_{t, T_{2}}^{(2)} Y_{t}^{(2)}+e^{-\kappa\left(T_{1}-t\right)} J_{t}\right)\right\}
\end{array}
$$

where $\bar{M}_{t, T}^{(i)}$ is defined in (12),

$$
\begin{aligned}
& A_{t, T}=u\left(A_{T, T_{1}}^{(1)}-A_{T, T_{2}}^{(2)}\right)+\int_{t}^{T} \lambda(s)\left(\varphi_{l}\left(u e^{-\kappa\left(T_{1}-s\right)}\right)-1\right) d s \\
& +u\left[-\bar{\alpha}_{1} \bar{\phi}_{1} \bar{\gamma}_{1} e^{-\bar{\alpha}_{1}\left(T_{1}-T\right)} h\left(t, T ; \bar{\alpha}_{1}\right)+\bar{\alpha}_{2} \bar{\phi}_{2} \bar{\gamma}_{2} e^{-\bar{\alpha}_{2}\left(T_{2}-T\right)} h\left(t, T ; \bar{\alpha}_{2}\right)\right. \\
& -\left(\bar{\gamma}_{2} \sigma_{Y}^{(2)}\right)^{2} e^{-2 \bar{\alpha}_{2}\left(T_{2}-T\right)} h\left(t, T ; 2 \bar{\alpha}_{2}\right)+\bar{\alpha}_{1} \bar{\phi}_{1} \bar{\gamma}_{1} e^{-\bar{\beta}_{1}\left(T_{1}-T\right)} h\left(t, T ; \bar{\beta}_{1}\right) \\
& -\left\{\left(\sigma_{X}^{(2)}\right)^{2}+2 \rho_{2} \bar{\gamma}_{2} \sigma_{X}^{(2)} \sigma_{Y}^{(2)}+\left(\bar{\gamma}_{2} \sigma_{Y}^{(2)}\right)^{2}\right\} e^{-2 \bar{\beta}_{2}\left(T_{2}-T\right)} h\left(t, T ; 2 \bar{\beta}_{2}\right) \\
& -\bar{\alpha}_{2} \bar{\phi}_{2} \bar{\gamma}_{2} e^{-\bar{\beta}_{2}\left(T_{2}-T\right)} h\left(t, T ; \bar{\beta}_{2}\right) \\
& -\rho_{1} \rho_{12} \bar{\gamma}_{1} \sigma_{X}^{(2)} \sigma_{Y}^{(1)} e^{-\bar{\alpha}_{1}\left(T_{1}-T\right)-\bar{\beta}_{2}\left(T_{2}-T\right)} h\left(t, T ; \bar{\alpha}_{1}+\bar{\beta}_{2}\right) \\
& +\left\{2 \rho_{2} \bar{\gamma}_{2} \sigma_{X}^{(2)} \sigma_{Y}^{(2)}+2\left(\bar{\gamma}_{2} \sigma_{Y}^{(2)}\right)^{2}\right\} e^{-\left(\bar{\alpha}_{2}+\bar{\beta}_{2}\right)\left(T_{2}-T\right)} h\left(t, T ; \bar{\alpha}_{2}+\bar{\beta}_{2}\right) \\
& \left.+\left\{\rho_{12} \sigma_{X}^{(1)} \sigma_{X}^{(2)}+\rho_{1} \rho_{12} \bar{\gamma}_{1} \sigma_{X}^{(2)} \sigma_{Y}^{(1)}\right\} e^{-\bar{\beta}_{1}\left(T_{1}-T\right)-\bar{\beta}_{2}\left(T_{2}-T\right)} h\left(t, T ; \bar{\beta}_{1}+\bar{\beta}_{2}\right)\right] \\
& +u^{2}\left[\frac{1}{2}\left(\bar{\gamma}_{1} \sigma_{Y}^{(1)}\right)^{2} e^{-2 \bar{\alpha}_{1}\left(T_{1}-T\right)} h\left(t, T ; 2 \bar{\alpha}_{1}\right)+\frac{1}{2}\left(\bar{\gamma}_{2} \sigma_{Y}^{(2)}\right)^{2} e^{-2 \bar{\alpha}_{2}\left(T_{2}-T\right)} h\left(t, T ; 2 \bar{\alpha}_{2}\right)\right. \\
& +\left\{\frac{1}{2}\left(\bar{\gamma}_{1} \sigma_{Y}^{(1)}\right)^{2}+\frac{1}{2}\left(\sigma_{X}^{(1)}\right)^{2}+\rho_{1} \bar{\gamma}_{1} \sigma_{X}^{(1)} \sigma_{Y}^{(1)}\right\} e^{-2 \bar{\beta}_{1}\left(T_{1}-T\right)} h\left(t, T ; 2 \bar{\beta}_{1}\right) \\
& +\left\{\frac{1}{2}\left(\bar{\gamma}_{2} \sigma_{Y}^{(2)}\right)^{2}+\frac{1}{2}\left(\sigma_{X}^{(2)}\right)^{2}+\rho_{2} \bar{\gamma}_{2} \sigma_{X}^{(2)} \sigma_{Y}^{(2)}\right\} e^{-2 \bar{\beta}_{2}\left(T_{2}-T\right)} h\left(t, T ; 2 \bar{\beta}_{2}\right) \\
& -\left\{\left(\bar{\gamma}_{1} \sigma_{Y}^{(1)}\right)^{2}+\rho_{1} \bar{\gamma}_{1} \sigma_{X}^{(1)} \sigma_{Y}^{(1)}\right\} e^{-\left(\bar{\alpha}_{1}+\bar{\beta}_{1}\right)\left(T_{1}-T\right)} h\left(t, T ; \bar{\alpha}_{1}+\bar{\beta}_{1}\right)
\end{aligned}
$$




$$
\begin{aligned}
& -\left\{\rho_{2} \bar{\gamma}_{2} \sigma_{X}^{(2)} \sigma_{Y}^{(2)}+\left(\bar{\gamma}_{2} \sigma_{Y}^{(2)}\right)^{2}\right\} e^{-\left(\bar{\alpha}_{2}+\bar{\beta}_{2}\right)\left(T_{2}-T\right)} h\left(t, T ; \bar{\alpha}_{2}+\bar{\beta}_{2}\right) \\
& \left.-\rho_{12} \sigma_{X}^{(1)} \sigma_{X}^{(2)} e^{-\bar{\beta}_{1}\left(T_{1}-T\right)-\bar{\beta}_{2}\left(T_{2}-T\right)} h\left(t, T ; \bar{\beta}_{1}+\bar{\beta}_{2}\right)\right]
\end{aligned}
$$

$\varphi_{l}(u)$ is the MGF of the individual jump sizes (see (75)), and the function $h(t, T ; \cdot)$ is given in equation (28).

Now that the transform is explicit, it is possible to use standard Fourier analysis techniques to value the spread option. Under some mild assumptions on the m.g.f. of jump sizes, it is possible to analytically continue the m.g.f. to the entire complex plane.

For completeness in the exposition, we remind the reader how the pricing equation (76) appears in Fourier transformed variables. Firstly,

$\Pi_{t, T}^{F}=P(t, T) F_{t, T_{2}}^{(2)} \mathbb{E}_{t}^{\widetilde{\mathbb{Q}}}\left[\left(F_{T ; T_{1}, T_{2}}-\alpha\right)_{+}\right]=P(t, T) e^{\bar{\alpha}} F_{t, T_{2}}^{(2)} \mathbb{E}_{t}^{\widetilde{\mathbb{Q}}}\left[\left(e^{Z_{T}-\bar{\alpha}}-1\right)_{+}\right]$,

where $\bar{\alpha}:=\ln (\alpha)$. By introducing $\eta(x):=\left(e^{x}-1\right)_{+}$, the expectation in equation (85) reduces to the product of Fourier transforms

$\mathbb{E}_{t}^{\widetilde{\mathbb{Q}}}\left[\left(e^{Z_{T}-\bar{\alpha}}-1\right)_{+}\right]=\frac{1}{2 \pi} \int_{-\infty}^{\infty} \tilde{\eta}(-p) \tilde{f}_{Z_{T}-\bar{\alpha}}(p) d p$,

where $\tilde{\eta}(p)$ and $\tilde{f}_{Z_{T}-\bar{\alpha}}(p)$ are the Fourier transforms of $\eta(x)$ and the probability density of $Z_{T}-\bar{\alpha}$, respectively. It is well known that

$\tilde{\eta}(p):=\int_{-\infty}^{\infty} e^{i p x} \eta(x) d x=\frac{1}{p(i-p)}$

whenever $\Im(p)>1$. A simple change of variables reveals that

$\tilde{f}_{Z_{T}-\bar{\alpha}}(p):=\int_{-\infty}^{\infty} e^{i p x} f_{Z_{T}-\bar{\alpha}}(x) d x=\mathbb{E}_{t}^{\widetilde{\mathbb{Q}}}\left[e^{i p\left(Z_{T}-\bar{\alpha}\right)}\right]=e^{-i \bar{\alpha} p} \Psi_{t}^{Z_{T}}(i p)$.

Putting these results together leads to our final pricing equation - up to a numerical integration.

Proposition 4.3 The price at time $t$ of the exchange option is

$\Pi_{t, T}=P(t, T) e^{\bar{\alpha}} F_{t, T_{2}}^{(2)} \int_{-\infty}^{\infty} \frac{e^{-i \bar{\alpha} p} \Psi_{t}^{Z_{T}}(i p)}{-p(p+i)} \frac{d p}{2 \pi}$,

with $\Psi_{t}^{Z_{T}}(\cdot)$ as in Proposition 4.2.

Some final remarks are crucial at this point:

1. In our framework, the price process of a spot exchange option is simply given by setting $T=T_{1}=T_{2}$ in equation (89).

2. The integral part of equation (89) seems formidable; however, the coefficients are nothing more complicated than exponentials and there exists very efficient numerical methods, such as FFT, for performing the integrals. Therefore, we do not pursue this further, and instead refer the reader to the monograph by Cont and Tankov (2004) for further information and references on these topics.

3. The market reveals the entire forward curve and, of course, the risk-free zero coupon bond prices. Before using the valuation formula, the model must be calibrated to these market prices. Once the parameters are calibrated, then the pricing equation (89) will provide consistent no-arbitrage prices to the various spread options. 
4. It is possible to repeat this analysis when both assets contain jumps. Needless to say, the resulting equations will be bulkier (but not fundamentally more complicated), and although the change of measure will be more subtle, it posses no real problems. However, in real applications, both assets typically do not contain sudden jumps, as one is usually the raw commodity used to produce electricity.

5. Some care must be taken to ensure the integration path in (89) remains in the intersection of the regions $\Im(p)>1$ and where the complex continuation of the function $\Psi_{t}^{Z_{T}}(z)$ is analytic in $z$. However, for typical jump distributions, such as double exponential and normal, $\Psi_{t}^{Z_{T}}(z)$ will be analytic in the region $\Im(p)>1$, and any simple path lying in $\Im(p)>1$ will do.

\section{Model Calibration}

In this section, we finally address the issue of parameter estimation. We perform this last step in two stages. Firstly, in Section 5.1 we provide a detailed review of an efficient method for calibrating the pure diffusion two-factor model to market futures prices, resulting in the risk-neutral model parameters. We also describe how jump parameters can be simultaneously estimated from market spot prices. Secondly, we describe how a method borrowed from interest rate model calibration can be used to estimate the real-world model parameters from a knowledge of spot and future prices. This simultaneous calibration of futures and spot prices to the risk-neutral and real-world measures further allows us to extract the implied market prices of risk. An alternative approach to real-world calibration is to use a well known Kalman Filter approach. Such approaches do not utilize futures prices data and can be quite useful. For more details on the calibration of various two-factor models to spot data and further references on the topic we refer to the work of Barlow, Gusev, and Lai (2004). Section 5.2 concludes with concrete applications of our statistical methodology to the NYMEX Light Sweet Crude Oil data and some further comments.

\subsection{Methodology}

Before proceeding to the calibration process, recall that the log of the forward price associated with the spot $S_{t}^{(i)}:=\exp \left\{g_{t}^{(i)}+X_{t}^{(i)}\right\}$ is given by (Section 3.2):

$$
\begin{aligned}
\log F_{t, T}^{(i)} & =g_{T}^{(i)}+\bar{G}_{t, T}^{(i)}+\bar{R}_{t, T}^{(i)}+e^{-\bar{\beta}_{i}(T-t)} X_{t}^{(i)}+\bar{M}_{t, T}^{(i)} Y_{t}^{(i)} \\
& =g_{T}^{(i)}+\bar{G}_{t, T}^{(i)}+\bar{R}_{t, T}^{(i)}+e^{-\bar{\beta}_{i}(T-t)}\left(\log S_{t}^{(i)}-g_{t}^{(i)}\right)+\bar{M}_{t, T}^{(i)} Y_{t}^{(i)} \\
& :=\bar{U}_{t, T}^{(i)}+\bar{M}_{t, T}^{(i)} Y_{t}^{(i)} .
\end{aligned}
$$

Here, the function $\bar{U}_{t, T}^{(i)}$ is introduced to simplify notation. Given the spot price data at time $t, \bar{U}_{t, T}^{(i)}$ is completely determined, while the last term $\bar{M}_{t, T}^{(i)} Y_{t}^{(i)}$ depends on the the hidden long-run mean level $Y_{t}$. Therefore, a standard nonlinear least-squares optimization cannot be applied directly. Instead, we will express the hidden factor in terms of the remaining model parameters and obtain an optimal fit to the observed futures curve at various time points.

Let $F_{t_{p}, T_{q}^{p}}^{(i) *}$ denote the observed futures prices at $t_{p} \in\left\{t_{1}, \ldots, t_{m}\right\}$ with delivery time $T_{q}^{p} \in\left\{T_{1}^{p}, \ldots, T_{n_{p}}^{p}\right\}$ and denote by $\bar{\Theta}$ a point in the (risk-neutral) parameter space $\bar{\Omega}$ of our model. For each given quoted times $t_{p}$, obtain $Y_{t_{p}}^{(i)}(\bar{\Theta})$ (as a function of the remaining parameters) such that it minimizes the following sum of squares:

$\operatorname{Sum}\left(t_{p}, \bar{\Theta}\right):=\sum_{q=1}^{n_{p}}\left[\log F_{t_{p}, T_{q}^{p}}^{(i)}-\log F_{t_{p}, T_{q}^{p}}^{(i) *}\right]^{2}$. 
The optimal $Y_{t_{p}}^{(i)}(\bar{\Theta})$ is easily found to be

$Y_{t_{p}}^{\#(i)}(\bar{\Theta})=\frac{\sum_{q=1}^{n_{p}}\left[\bar{M}_{t_{p}, T_{q}^{p}}^{(i)}\left(\log F_{t_{p}, T_{q}^{p}}^{(i) *}-\bar{U}_{t_{p}, T_{q}^{p}}^{(i)}\right)\right]}{\sum_{q=1}^{n_{p}}\left[\bar{M}_{t_{p}, T_{q}^{p}}^{(i)}\right]^{2}}$.

Substituting this optimal value into the initial sum of squares (93), summing over the range of initial times $\left\{t_{p}\right\}$ and performing a nonlinear least-squares optimization as follows:

$\bar{\Theta}^{*}:=\operatorname{ArgMin}_{\bar{\Theta} \in \bar{\Omega}} \sum_{p=1}^{m} \sum_{q=1}^{n_{m}}\left[\bar{U}_{t_{p}, T_{q}^{p}}^{(i)}+\bar{M}_{t_{p}, T_{q}^{p}}^{(i)} \cdot Y_{t_{p}}^{\#(i)}(\bar{\Theta})-\log F_{t_{p}, T_{q}^{p}}^{(i) *}\right]^{2}$,

provides an "optimal fit" of the model to futures prices, therefore obtaining our risk-neutral model parameters $\left(\bar{\beta}, \bar{\alpha}, \bar{\phi}, \sigma_{X}, \sigma_{Y}, \rho\right)$. An implementation of this methodology naturally requires both futures prices and spot prices at the corresponding futures quote times.

It is worth mentioning that this method does not directly extend to jump-diffusion (spot) models since the coefficients of the $X_{t}$ and $J_{t}$ terms in the forward price (73) are unequal. This prevents a simple factorization into functions that are known given the spot prices and the hidden process $Y_{t}^{(1)}$. To circumvent that problem a standard alternative methodology is to extract the jump parameters from the spot price data only. Such a calibration can be carried out in two ways: (i) by cutting off all data points lying below a given level, so that only spikes remains. From these data points one can then infer the value of the various jump parameters (see for example the discussion in Clewlow and Strickland (2000)); or (ii) by utilizing particle-filter approaches which generalizes the Kalman filter to non-normal innovations (see for example Aiube, Baidya, and Tito (2005)). The standard assumption that the calibrated jump parameters are unchanged when moving to the risk-neutral world is then invoked. Given, the jump parameters it is now possible to repeat the previous futures price calibration process to obtain the riskneutral diffusive components.

We now turn to the real-world $\mathbb{P}$-parameters $\left(\beta, \alpha, \phi, \sigma_{X}, \sigma_{Y}, \rho\right)$ estimation problem. Since under any diffusive model for spot prices, a change of measure from the real-world to risk-neutral cannot alter the volatility structure of the model, from equation (95) we obtain $\sigma_{X}, \sigma_{Y}$ and $\rho$ under $\mathbb{P}$. The remaining set of $\mathbb{P}$-parameters $(\beta, \alpha, \phi)$ are relatively straightforward to obtain. Firstly, we obtain $\beta$ and $\phi$ via linear regression on the spot price data assuming a mean reverting one-factor model for $X_{t}$ as a proxy to our two-factor model. The one-factor mean-reversion level $\phi$ becomes, in our model, the stochastic long-run mean level $Y_{t}$. Secondly, we perform a similar regression on the estimated hidden process $Y_{t}^{\#}$ which was obtained by minimizing the error on an individual futures curve basis (see equation (94)). Equation (94) provides a data set which we can use as an input in a regression to find $\alpha$. We find this procedure to be very stable and, as shown in the next section, leads to reliable parameter estimation.

\subsection{Some Results: Crude Oil}

\begin{tabular}{rrrrrrrrr}
\hline \hline $\bar{\alpha}$ & $\bar{\beta}$ & $\bar{\phi}$ & $\alpha$ & $\beta$ & $\phi$ & $\sigma_{X}$ & $\sigma_{Y}$ & $\rho$ \\
0.15 & 0.31 & 3.27 & 0.73 & 1.07 & 4.21 & $33 \%$ & $63 \%$ & -0.96 \\
\hline \hline
\end{tabular}

Table 1

The calibrated real-world and risk-neutral model parameters using the NYMEX Light Sweet Crude Oil spot and futures data for the period 1/10/2003 - 25/07/2006.

In this section, we present the calibration results of our two-factor pure diffusion model (Section 3) to the NYMEX Light Sweet Crude Oil spot and futures data for the period 1/10/2003 to 25/07/2006. In 
Table 1, we report the calibration results for the real-world and risk-neutral parameters. There are a few notable observations: (i) both real-world mean-reversion rates $\alpha$ and $\beta$ are significantly larger than the risk-neutral mean-reversion rates $\bar{\alpha}$ and $\bar{\beta}$, (ii) The real-world long-run mean-reversion level $\phi$ is larger than the risk-neutral long-run mean $\bar{\phi}$, and (iii) in both the real-world and risk-neutral measures, the mean-reversion rates $(\alpha$ and $\bar{\alpha})$ of the long-run mean $Y_{t}$ are smaller than the mean-reversion rates ( $\beta$ and $\bar{\beta})$ of the log-spot $X_{t}$.

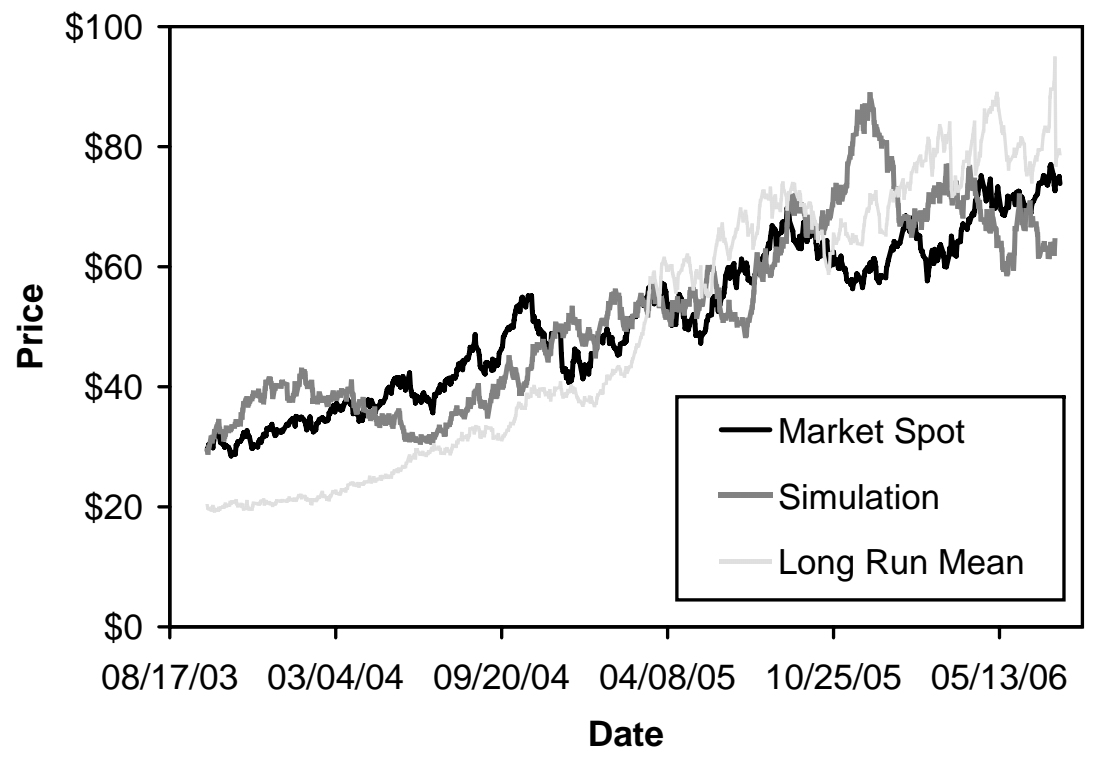

Figure 1. The NYMEX Light Sweet Crude Oil spot prices and simulated spot prices based on the calibration in Table 1.

In Figure 1, we plot the spot price data together with the stochastic long-run mean level $Y_{t}^{\#}$ implied by the futures prices. For comparison, we also include one simulated sample path based on a simulation of the prices using the real-world model parameters in Table 1. Figure 2 illustrates the relative root-mean squared-error (RMSE) for each forward curve using the model parameters reported in Table 1. The average RMSE per curve is $0.7 \%$ with only a few dates having relative errors larger than $1 \%$. Recall that the model parameters are fixed over all curves, and are not adjusted on a curve by curve basis. With this in mind, we believe the fit is excellent.

We also investigated the stability of our estimation procedure through time. We calibrated the model to the first $T_{1}$ calender days and then to the first $T_{2}$ calender days and so on. The time periods are approximately equally spaced at 88 days from $1 / 10 / 2003$ to $25 / 07 / 2006$. We report these calibration results in Table 2. The most stable parameters are the volatility $\sigma_{X}$ of the $X_{t}$ process, the volatility $\sigma_{Y}$ of the stochastic long-run mean level $Y_{t}$, the mean-reversion level $\bar{\alpha}$ of the stochastic long-run mean $Y_{t}$ and the correlation coefficient $\rho$. The remaining parameters, although not as unvarying as the previous four, are well behaved. None of the parameters suddenly explode or tend to zero, and always remain realistic.

Finally, since we were successful in extracting the real-world and risk-neutral parameters, we further extract the implied market prices of risk through Theorem 3.2. The evolution of the implied market prices of risk $\lambda$ and $\Psi$ are displayed in Figure 3. Interestingly, they are very strongly correlated to one another, becoming almost indistinguishable after one and a half years. This is due to the high correlation coefficient of $\rho=-0.96$. Also, both market prices of risk are negative for essentially the entire time 


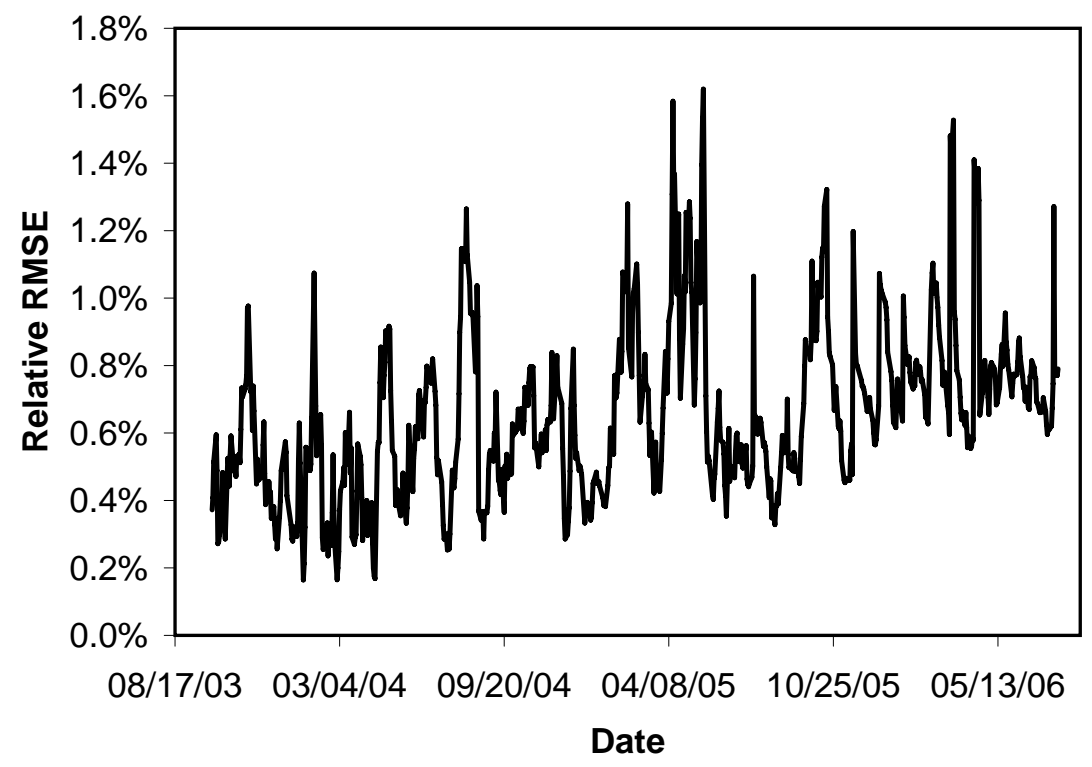

Figure 2. The relative root-mean squared-error of each forward curve based on the calibration in Table 1.

period. This is a reflection of the real-world mean-reversion rates $(\alpha$ and $\beta)$ and real-world long run mean-reversion level $(\phi)$ being higher than the risk-neutral ones $((\bar{\alpha}, \bar{\beta}$ and $\bar{\phi}))$. The market therefore attaches slower reversion rates and lower long run levels than the implied historical levels.

\section{Conclusions}

We introduced a diffusive two-factor mean-reverting process for modeling spot prices of energy commodities. The two-factor diffusive model extends the one-factor mean-reverting model by making the long-run mean a stochastic degree of freedom which itself mean-reverts to a specified level. We also generalized the model to incorporate jumps in the price process such as those observed in electricity prices. To maintain realistic mean-reversion rates and diffusive volatilities we decoupled the jump and diffusive processes. Given our affine modeling framework, we were successful in obtaining expressions for the forward price curves in terms of elementary functions. Through a measure changed induced by the forward price process, our modeling framework allows us to obtain closed form pricing equations for various spread options. We obtained pricing equations under both an Actuarial and risk-neutral valuation procedures.

Finally, we provided a method for calibrating both the diffusion and jump-diffusion models to spot and forward prices simultaneously. This simultaneous calibration procedure further allowed us to extract the implied market prices of risk. Using the NYMEX light sweet crude oil data set, we demonstrated that the calibration procedure produces realistic and stable implied risk-neutral and real-world model parameters.

\section{Acknowledgements}

The authors would like to thank Bill Bobey for assistance with acquiring the NYMEX data and Hans Tuenter for fruitful discussions on various aspects of the energy markets. 
Table 2

\begin{tabular}{rrrrrrr}
\hline \hline \# Days & $\bar{\beta}$ & $\bar{\alpha}$ & $\bar{\phi}$ & $\sigma_{X}$ & $\sigma_{Y}$ & $\rho$ \\
\hline 88 & 0.38 & 0.26 & 3.34 & $33 \%$ & $19 \%$ & -0.97 \\
176 & 0.52 & 0.21 & 3.06 & $33 \%$ & $54 \%$ & -0.79 \\
264 & 0.62 & 0.10 & 2.36 & $33 \%$ & $56 \%$ & -0.73 \\
352 & 0.61 & 0.08 & 1.97 & $35 \%$ & $60 \%$ & -0.64 \\
440 & 0.52 & 0.09 & 2.33 & $35 \%$ & $58 \%$ & -0.71 \\
528 & 0.43 & 0.10 & 2.98 & $34 \%$ & $52 \%$ & -0.95 \\
616 & 0.34 & 0.13 & 3.24 & $34 \%$ & $58 \%$ & -0.96 \\
704 & 0.31 & 0.15 & 3.27 & $33 \%$ & $63 \%$ & -0.96 \\
\hline Average: & 0.48 & 0.12 & 2.74 & $34 \%$ & $57 \%$ & -0.82 \\
Stdev: & 0.13 & 0.05 & 0.52 & $1 \%$ & $4 \%$ & 0.14 \\
\hline \hline
\end{tabular}

This table shows the evolution of the estimated risk-neutral parameters through time as more recent data is added to the calibration procedure. The average and standard deviation are reported using 176 days onwards.

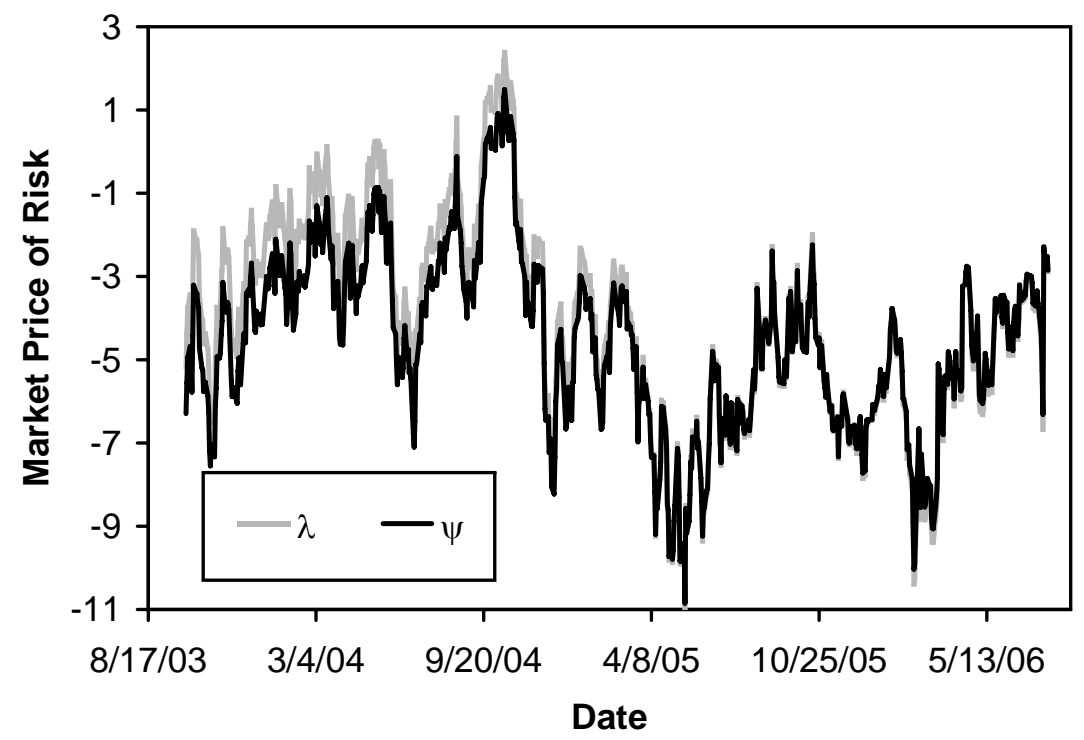

Figure 3. This diagram depicts the evolution of the implied market prices of risk using the calibrated real-world and risk-neutral parameters. 


\section{References}

Aiube, F., T. Baidya, and E. Tito, 2005, "Stochastic processes of commodity prices: a particle filter approach," Preprint.

Barlow, M., Y. Gusev, and M. Lai, 2004, "Calibration of Multifactor Models in Electricity Markets," International Journal of Theoretical and Applied Finance, 7, 101-120.

Carmona, R., and V. Durrleman, 2003, "Pricing and Hedging Spread Options," SIAM Review, 4, 627-685.

Carr, P., and D. Madan, 1999, "Option valuation using the fast Fourier transform," The Journal of Computational Finance, 2, 61-73.

Cartea, A., and M. Figueroa, 2005, "Pricing in Electricity Markets: a mean reverting jump diffusion model with seasonality," Applied Mathematical Finance, 22(4), 313-335.

Clewlow, L., and C. Strickland, 2000, Energy Derivatives: Pricing and Risk Management., Lacima Publications.

Cont, R., and P. Tankov, 2004, Financial Modelling with Jump Processes, Chapman and Hall.

Cortazar, G., and E. Schwartz, 1994, "The Valuation of Commodity Contingent Claims," The Journal of Derivatives, 1, 27-29.

Duffie, D., J. Pan, and K. Singleton, 2000, "Transform Analysis and Asset Pricing for Affine JumpDiffusions," Econometrica, 68, 1343-1376.

Eydeland, A., and K. Wolyniec, 2003, Energy and Power Risk Management, John Whiley and Sons.

Gibson, R., and E. Schwartz, 1990, "Stochastic Convenience Yield and the Pricing of Oil Contingent Claims," The Journal of Finance, 45, 959 - 976.

Harrison, J., and S. Pliska, 1981, "Martingales and stochastic integrals in the theory of continuous trading," Stochastic Process. Appl., 11(1), 215-260.

Hull, J., 2005, Options, Futures and Other Derivatives, Prentice Hall, 6th edn.

Margrabe, W., 1978, "The Value of an Option to Exchange One Asset for Another," The Journal of Finance, 33, 177-186.

Pilipovic, D., 1997, Valuing and Managing Energy Derivatives, New York, McGraw-Hill. 